\title{
A TIME-SERIES AND SPATIAL ANALYSIS OF 56 YEARS (1961-2017) OF RAINFALL HISTORICAL DATA FROM MALAYBALAY, BUKIDNON
}

\author{
B. J. Buenaobra ${ }^{1}$, MK L. Alleto $^{2}$, JM V. Manhuyod ${ }^{1}$ \\ ${ }^{1}$ Plantation Geomatics Dept., Del Monte Philippines Inc., Agusan Canyon, Manolo Fortich, \\ 2 Crop Production Unit, Del Monte Philippines Inc., Agusan Canyon, Manolo Fortich, \\ Bukidnon, Northern Mindanao - (buenaobrabj, alletoml, manhuyodjmv) @delmonte-phil.com
}

KEYWORDS: Time Series Analysis, Rainfall Density, Rainfall Seasonality, Autocorrelation, Stationarity

\begin{abstract}
:
This paper focuses on using time series and spatial analysis methods to detect climate change indicators in Malaybalay, Bukidnon. We look at 56 years of historical rainfall data between the years 1961 to 2017 and perform a computational method for data processing to arrive at spatial statistics and provide data visualization. We demonstrate the use of the Augmented Dickey-Fuller test (ADF), where a $p$-value is tested versus a threshold to reject or accept the null hypothesis for a stationarity test. For the seasonality test, we perform a time-domain signal processing by an autocorrelation function. The time-series analysis shows that for Malaybalay, Bukidnon rainfall data shows ADF statistic of -16.348964 , a $p$-value $=0.000000$ with critical values $1 \%:-3.431,5 \%:-2.862,10 \%:-2.567$. Hence, the significant negative values indicate more likely to reject the null hypothesis. We showed that rainfall does not demonstrate periodicity, is not seasonal, and is non-stationary. This work does not cover those that can be detected and attributed to anthropogenic causes.
\end{abstract}

\section{INTRODUCTION}

\subsection{Malayblay, Bukidnon as a region of interest}

In the definition we find from the United States Geological Survey (USGS), the terms Global Warming and Climate Change are well differentiated. In verbatim, "Global warming” refers to the rise in global temperatures due mainly to the increasing concentrations of greenhouse gases in the atmosphere. "Climate change" refers to the growing changes in climate measures over a long period - including precipitation, temperature, and wind patterns. According to the Department of Science and Technology (DOST), the Philippines' oldest weather station system and weather data are located in Malaybalay, Bukidnon. The resolution specified by the agency is $50 \mathrm{~km}$.

To describe our area of interest in Region 10, Malaybalay City in Northern Mindanao has a total land area of 96,919 hectares, about $9.23 \%$ of the total area of Bukidnon province. An estimate of $65 \%$ is forestland/timberland, and the remaining $35 \%$ are alienable and disposable areas for purposes such as agriculture or industry. (PhilAtlas, 2021)

\section{COMPUTATIONAL EXPERIMENT SETUP}

\subsection{Rainfall Data Pre-Processing}

The rainfall data from the years 1961-2017 of Malaybalay, Bukidnon was purchased at the cost of Php.1.0 per parameter per day acquisition from the national weather bureau from CADPAGASA offices at the beginning of 2018. It was delivered in M.S. Excel format in 2 columns (dates) by 20,805 rows of rainfall data. (DOST PAGASA, 2021)

Marking of " $T$ " designated no data as those "0" were considered no rainfall on the corresponding dates. As in the usual practice of data pre-processing, unknown data entries are removed or replaced by data transformation numerical operations such as averaging. Data with “T” markings were replaced by the average from their immediate neighboring cells value. It is assumed that no instrumental error was caused by the weather station instrument when the data of "0" appeared on the cells. The entire data processing and visualization were done Python programming using Jupyter Notebooks within the Anaconda development environment. (Anaconda Analytics, 2021). We demonstrate the plot generation programmatically in Python in the Appendices. (Anaconda, 2021)

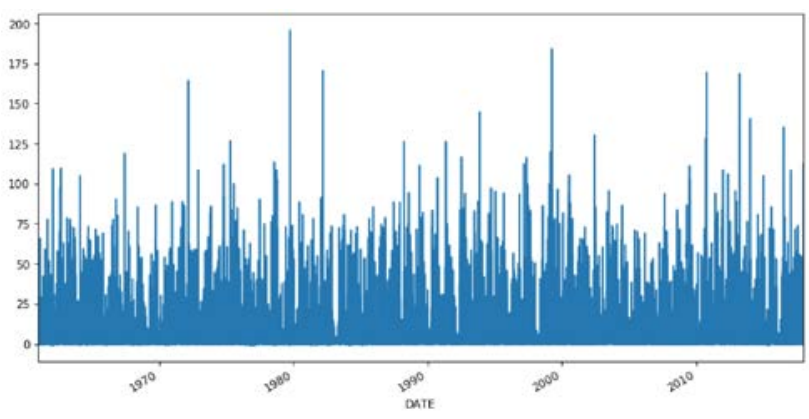

Figure 1. Line plot of Malaybalay rainfall between 1961-2017

\subsection{General observations from January 1961 to 2017}

For the same months but 56 years apart of rainfall, the later dates show more rain and higher intensity rain events. To site, on the16th January and 18th in 2017 is rainfall of $83.3 \mathrm{~mm}$ and $108.4 \mathrm{~mm}$ that when compared in 1961 on the same month and day there was only $0.0 \mathrm{~mm}$ and $1.5 \mathrm{~mm}$. In 1961 the highest rainfall occurred on 29 January at $29.5 \mathrm{~mm}$, while for 2017 , it was $108.4 \mathrm{~mm}$ on 8 January. These were followed by more minor but still more intense than those occurring in 1961 on 28 January at $49.8 \mathrm{~mm}$ and $74.3 \mathrm{~mm}$. Typhoons visit the island of Mindanao as shown by experience during the last quarter to the first quarter of the new year. (DOST PAGASA, 2021) 


\subsection{Descriptive Statistics of 1961-2017 Rainfall data}

Calculating the descriptive statistics for the spanned years of the natural rainfall data from Malaybalay, Bukidnon, we have the following results from programmatic data processing:

\begin{tabular}{|l|c|}
\hline Name: Rainfall & dtype: float64 \\
\hline count & 20805 \\
\hline mean & 7.127195 \\
\hline std & 13.995723 \\
\hline min & -1.000000 \\
\hline $25 \%$ & 0.000000 \\
\hline $50 \%$ & 1.000000 \\
\hline $75 \%$ & 7.800000 \\
\hline max & 195.900000 \\
\hline
\end{tabular}

Table 1. Descriptive Statistics Malaybalay Rainfall

We can detect practically twice as much deviation from the mean value for the entire 56 years of rainfall. Also, at least $3 / 4^{\text {th }}$ of rains as the whole is around $8 \mathrm{~mm}$ of rain, and minima occur with zero precipitation or dry periods. Probably the most decisive rain event could be as high as under $200 \mathrm{~mm}$. We demonstrate programmatically using Python, obtaining the descriptive statistics in the Appendices. (Anaconda, 2021)

\section{TIME-SERIES AND SPATIAL ANALYSIS}

\subsection{Temporal series of 56-year Malaybalay Rainfall}

For a rainfall analysis, both the amount of rain with their temporal component is taken into account. The dimension is in units of day rain for each year for all 56 years of the entire observation. We have shown from the previous section the basic descriptive statistics. We offer the plot of the amplitudes on successive stacks of rainfall variation with time to visualize them over several years. This is shown in Figure 1.

\subsection{Test for Stationarity}

When the observations in time series are not dependent on time, we say it is stationary; otherwise, it's called non-stationary. It is also a test if our observations are consistent given the temporal structure. The most basic statistical test is the determination of the means and variances for stationarity or non-stationarity. The mean values are practically the same. Hence, Malaybalay rainfall is therefore stationary. (Brownlee, J., 2021)

\begin{tabular}{|l|l|}
\hline mean1=6.943857 & mean2=7.334317 \\
\hline variance1=186.006267 & variance2=205.297065 \\
\hline
\end{tabular}

Table 2. Means and Variance results from splitting data

\subsection{Augmented Dickey-Fuller test for stationarity}

We also made use of the so-called Augmented Dickey-Fuller test (ADF). A p-value that results from the ADF test below a threshold suggests rejecting the null hypothesis, which implies observation is stationary. Otherwise, a p-value above the threshold indicates means we failed to reject the null hypothesis, and thus it is non-stationary. Performing the ADF program, we have the following results in Table 3 . The calculation is done programmatically from reading the measurement data using Python a code within the Jupyter Notebook (Augmented DickeyFuller Statistical Test). Calling the Autocorrelation function for the 1961-2017 Malaybalay, Rainfall data, we produced a plot in Figure 3.

\begin{tabular}{|l|l|}
\hline ADF Statistic & -16.348964 \\
\hline p-value & 0.000000 \\
\hline Critical Values & \\
\hline $1 \%$ & -3.431 \\
\hline $5 \%$ & -2.862 \\
\hline $10 \%$ & -2.567 \\
\hline
\end{tabular}

Table 3. ADF Test Results

The $p$-value $<=0.05$, we reject the null hypothesis $(\mathrm{H} 0)$; rainfall does not have a unit root; hence, it is stationary. It does not have a time-dependent structure. (Brownlee, J., 2021)

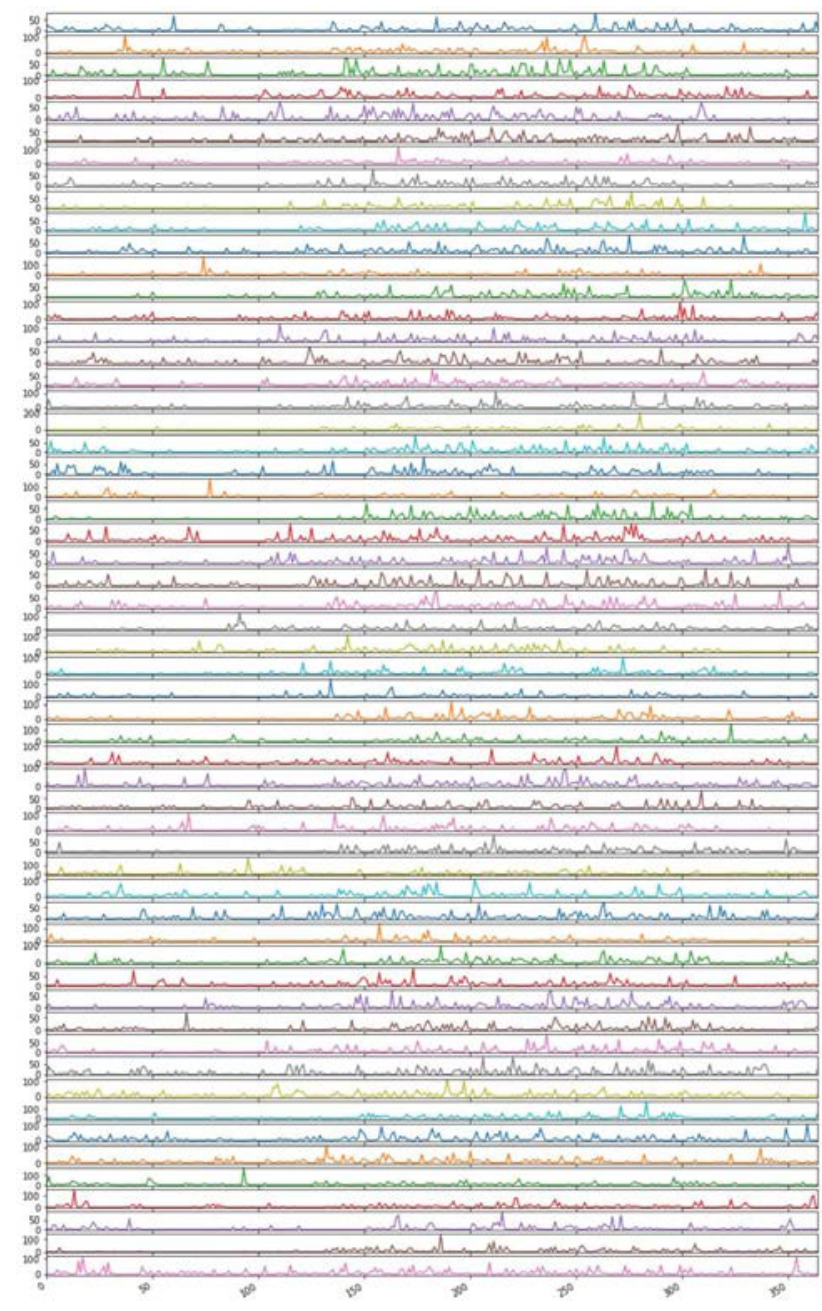

Figure 2. A stacked plot of Malaybalay Rainfall (1961-2017)

\subsection{Test for Seasonality and Periodicity}

For the test for seasonality, we perform the Autocorrelation function, also called self-correlation. When the time series data show statistically significant periodicity, it is seasonal.

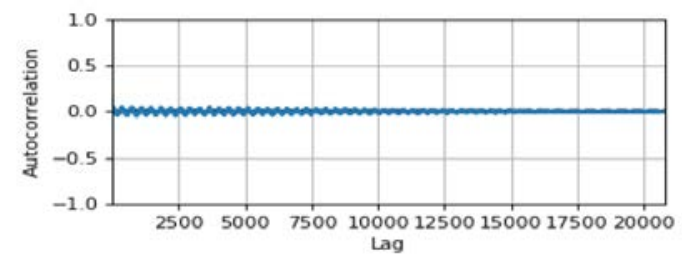

Figure 3. Autocorrelation of 1961-2017 Malaybalay rainfall 
We can infer no significant periodicity or cycle of the same intensity across all the years, indicating no seasonality. (Proakis J., Manolakis D.G, 1995)

\subsection{Spatial Visualization of 56-year Malaybalay Rainfall}

For a 2D visualization of rainfall, as shown in Figure 3. Essentially the plots are 2D representations of a matrix where a column is made up of day rows, another for intensity, and generated a Gaussian interpolated heat map. These can show the intensity peaks of the month's rainfall for every year.

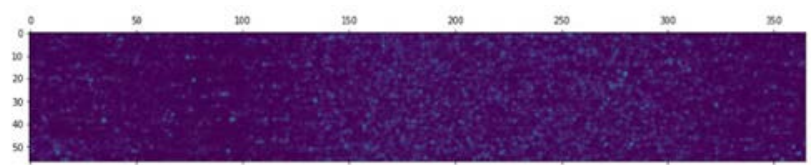

Figure 4. Heat Map of a typical yearly rainfall data

Figure 5 and Figure 6 below show the specific month data's heat map with Gaussian interpolation. Here we show the series for the years 1961 and 2017 below. Each column represents one month, with rows representing the days of the month from days 1 to 31 .

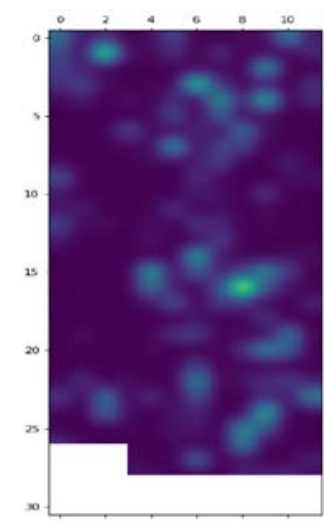

Figure 5. Heat Map 1961

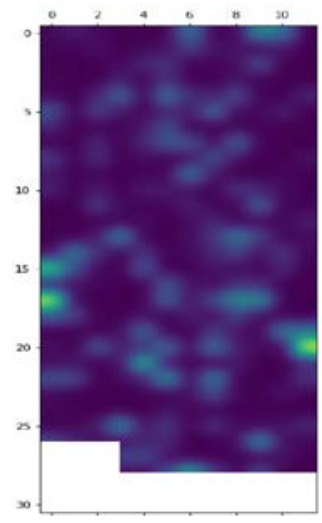

Figure 6. Heat Map 2017

We can further visualize the peaks of rainfall distribution in the 3D surface generation by image processing software, as shown in Figure 7 and Figure 8 (ImageJ)
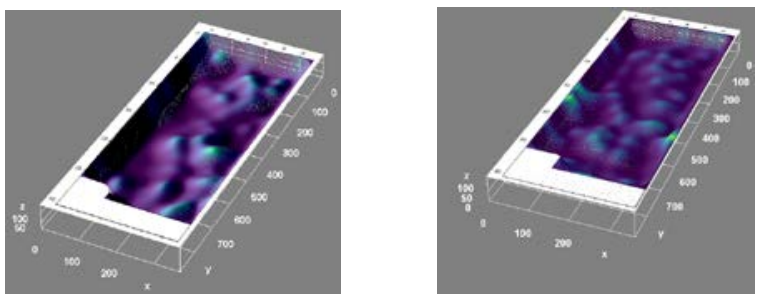

Figure 7. 3D surface plot 1961 Figure 8. 3D surface plot 2017

\subsection{Weather Station vs. Remote Sensed Dataset Quality}

The Malaybalay 56 years of rainfall of 1961-2017 acquired and continues to this day collected by the agency PAGASA-DOST in Region $\mathrm{X}$, is considered the oldest rainfall data obtained by the oldest weather station in the country. We are interested in comparing the old sensor on a mast in the ground with the newer satellite sensor over the atmosphere and extraterrestrial space. We have considered the data archive from LARC-NASA with its oldest available data as starting date. We have subsetted the years from January 1982 to December 2017 from the Malaybalay rainfall data dates to match the. We plot the histogram of the two datasets in Figure 9 below.

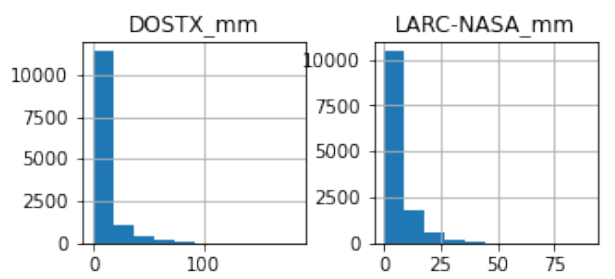

Figure 9. Histogram of rainfall dataset

We can see that the PAGASA-DOST rainfall histogram is practically the same as for frequency. However, the localized ground measurement data tends to spread more, reaching near $100 \mathrm{~mm}$, while LARC-NASA, which is remote sensed and is nearly $50 \mathrm{~mm}$ with the integration process. For the line plots, we show both rainfall datasets we depict the matching span of years for visual comparison in Figure 10 below.

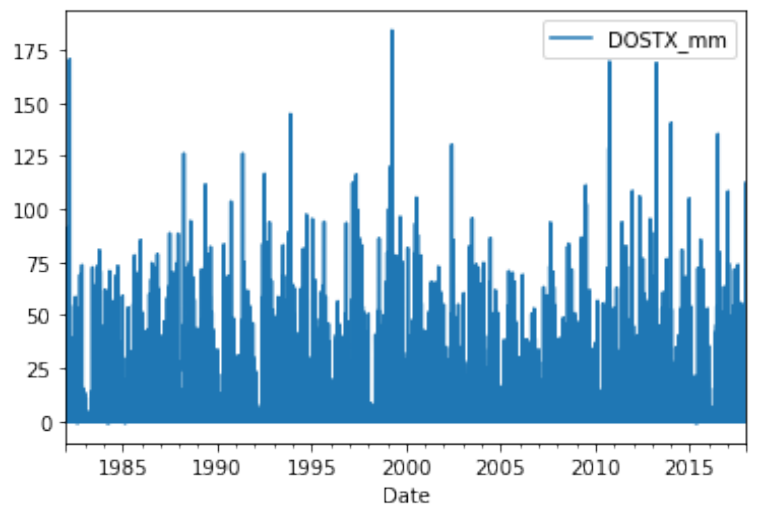

Figure 10. Weather Station Data PAGASA-DOST

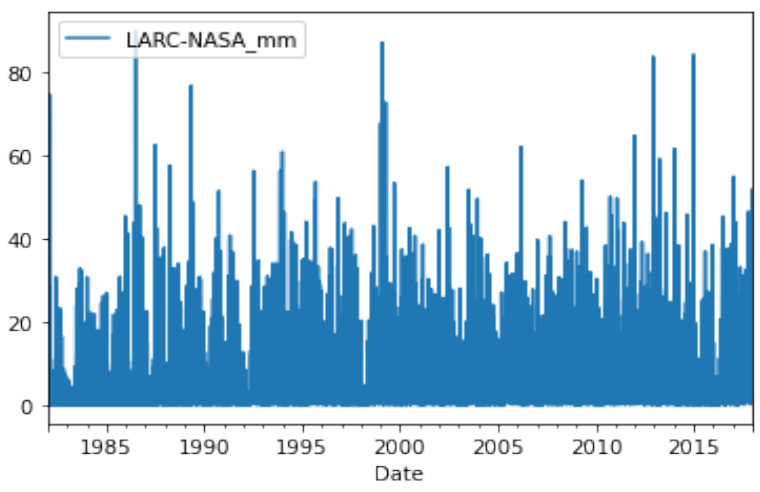

Figure 11. Remote Sensed Data LARC-NASA

We show the comparison of their individual shape of distribution by deriving their Density Plot programmatically from the two dataset. We can view the density plot as the smooth version of a histogram. In the following page we show the results for the density plot for Malaybalay historical rainfall data from PAGASA DOST collected by weather station and for a remote sensed data obtained from LARC-NASA archives. Figure 12 shows density plot for PAGASA-DOST dataset and followed by 
the Figure 13 that shows the density plot for LARC-NASA. The density plots below shows LARC-NASA data have narrower base than DOST dataset and with higher density.

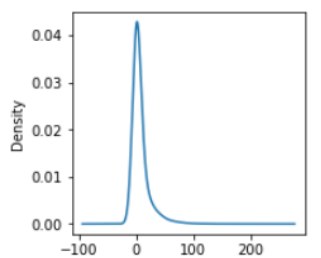

Figure 12. Density Plot DOST-PAGASA

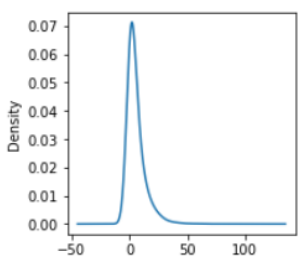

Figure 13. Density Plot LARC-NASA
We also performed the ADF Statistics for each dataset the results are shown below for each case.

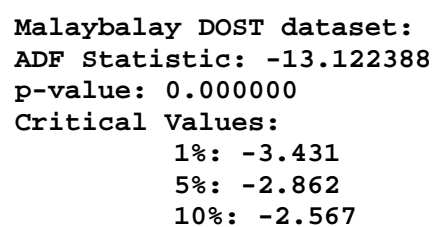

A p-value below a threshold (such as $5 \%$ or $1 \%$ ) suggests we reject the null hypothesis (stationary), otherwise, a p-value above the threshold suggests we fail to reject the null hypothesis (nonstationary).

We also perform the Stationarity test for each dataset looking at the results of the means and variance. The results shows are shown below.

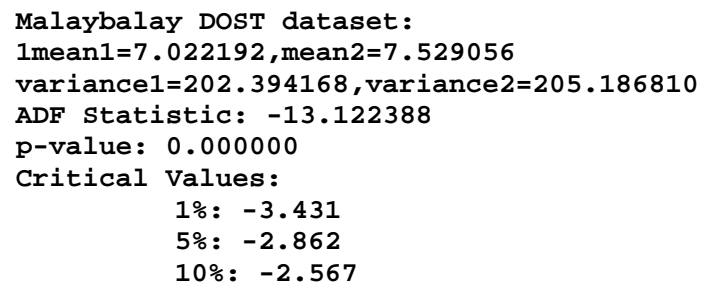

The mean values for Malaybalay are practically the same. Hence, Malaybalay rainfall is therefore stationary. There is a considerable difference in the mean values for the LARC-NASA dataset however, the p-values prevail indicative of it being stationary as well.

\section{CONCLUSIONS}

The result of computational tests of rainfall data of Malaybalay, Bukidnon, showed that the 56 years of rainfall is neither seasonal nor periodic. Furthermore, the examination of means and variance points to rain as being stationary. The visualization using a heat map shows no evidence of the same rainfall intensity from 1961 to 2017. Even with some indication of more significant rain in the later years that could reach under $200 \mathrm{~mm}$, it is not a strong case for a sign of climate change. The unavailability of the corresponding temperature on the same span of years as rainfall did permit the authors to investigate the temporal variation of temperature to support the cause of climate change detection.

\section{REFERENCES}

DOST, Department of Science and Technology, Philippine Atmospheric, Geophysical and Astronomical Services Administration

(https://www.pagasa.dost.gov.ph/climate/climate-data)

(20 October 2021)

Climate Change in the Philippines, DOST, Department of Science and Technology, Philippine Atmospheric, Geophysical and Astronomical Services Administration (https://www.pagasa.dost.gov.ph/information/climate-changein-the-philippines) (20 October 2021)

\section{PhilAtlas}

Malaybalay, Province of Bukidnon

(https://www.philatlas.com/mindanao/r10/bukidnon/malaybalay .html) (20 October 2021)

\section{Anaconda}

Python 3.8 64-bit (Anaconda Individual Edition Version) (https://www.anaconda.com/products/individual)

(18 October 2021)

Brownlee, J., 2021: Deep Learning for Time Series Forecasting (https://machinelearningmastery.com/deep-learning-for-timeseries-forecasting/) (20 October 2021)

Image

64-bit Java 1.8.0_172 (Platform Independent version) (https://imagej.nih.gov/ij/download.html ) (20 October 2021)

Proakis J., Manolakis D.G, 1995: Digital Signal Processing: Principle, Algorithms, and Applications. Prentice-Hall $3^{\text {rd }}$ Ed.

Data Access Viewer - NASA Power

(https://power.larc.nasa.gov/data-access-viewer/)

(6 November 2021)

\section{APPENDIX}

\section{Loading and checking Python modules}

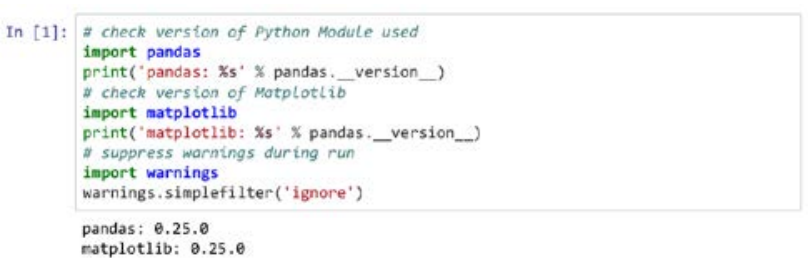




\section{Reading in rainfall data}

In [2]: from pandas import Series

from pandas import read_csv

series = readcsy('Malaypalay Daily_total-Rainfall_(1961-2017) Dataprep.csy', he ader $=\theta$, index_col $=\theta$,

print (series.head())

DATE

1961-01-e1 29.8

$\begin{array}{ll}1961-01-02 & 16.8 \\ 1961-01-03 & 14.2\end{array}$

1961- $81-04$ 10.7

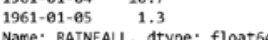

\section{Calculate the number of observations from data}

In [3]: \# sumarize the dimensions of a time series

from pandas import read_csv
series = read_csv('Malaybalay_da1ly_total-Rainfall_(1961-2017)Dataprep.csv', header $=\theta$, index_col $=\theta$, parse_dates $=$ True,

squeeze=True)

(5ize)

\section{Calculate descriptive statistics in Python}

In [6]: \# calculate descriptive statistics

from pandas import read_csv

series = read_csv('Malaybalay_Daily_Total-Rainfal1_(1961-2017)DataPrep.csv', squeeze=True)

squeeze=True)
print(series.describe())

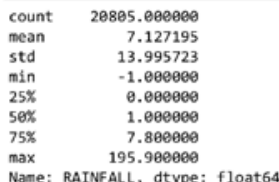

\section{Create the historical rainfall line plot}

In [7]: \# create a line plot

from pandas import read_csv

from matplotlib import pyplot

figure(num-None, figsize=(12, 6), dpi=80, facecolor=' $w$ ', edgecolor=' $k$ ')

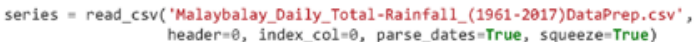

series.plot(

pyplot. show()

\section{Create stacked line plots of temporal yearly rainfall}

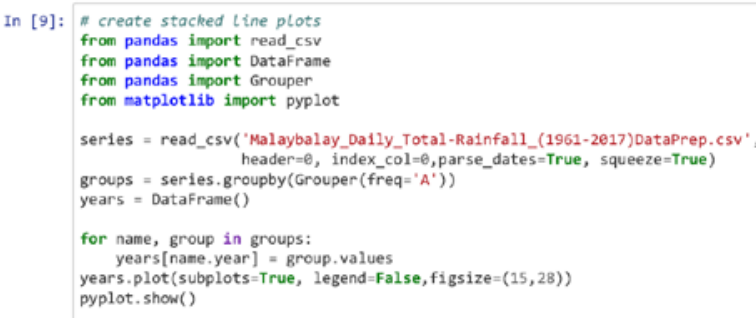

\section{Subsetting dates of interest from rainfall data}

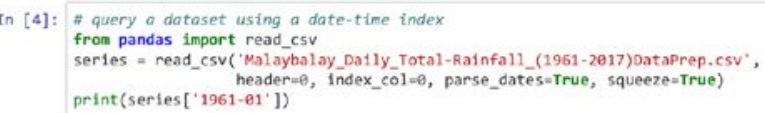

\section{Create a heat map of yealy rainfall data}

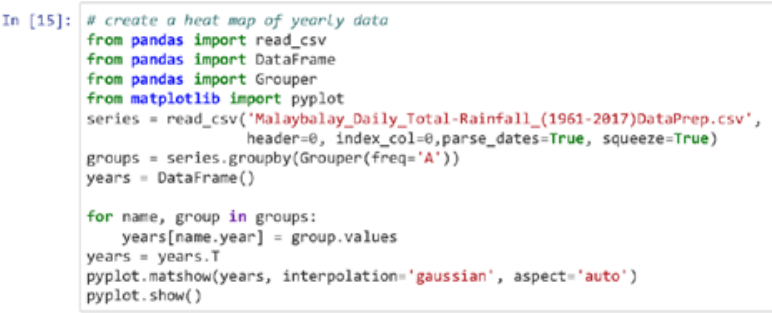

\section{Create a heat map of monthly rainfall data}

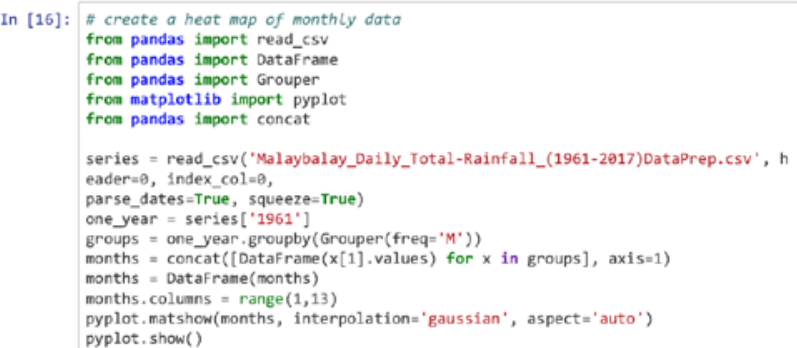

Test for Stationarity by Means and Variances

[13]: \# we split the rainfall data and compare their means and variances

series = read_csv ('Malaybalay_Daily_Total-Rainfall_(1961-2017) e365-Day.csv' $\mathrm{X}=$ series.values

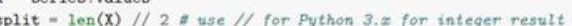

$\mathrm{x}_{1}, \mathrm{x}_{2}=\mathrm{x}[0:$ :sp $1 \mathrm{it}], \mathrm{x}[\mathrm{sp} 1 \mathrm{it}, \mathrm{s}]$

mean1, mean $2-x_{1} \cdot \operatorname{mean}(), x_{2} \cdot \operatorname{mean}()$

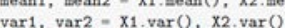

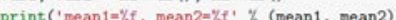

print ('variance 1- $\%$, , variance $2=\%$ ' ' $\%$ (var1, var2)

mean1-6.943857, mean2-7.334317

variance $1=186.006267$, variance $2=205.297065$

\section{Augmented Dickey-Fuller Statistical Test}

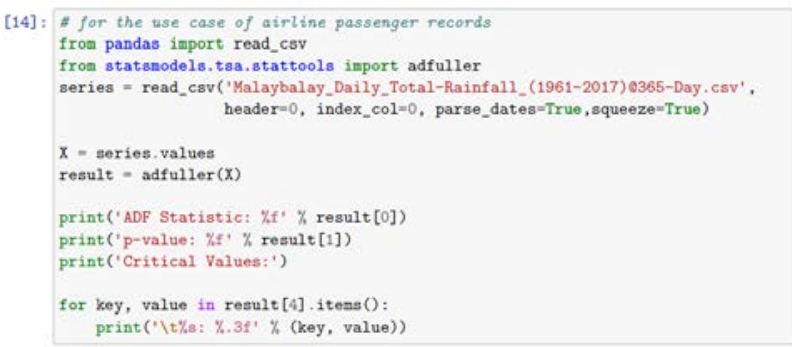

\section{Autocorrelation Function}

[47]: \# plot the autocorrelation of any weather variable input on the parenthesis from natplotlib.pyplot import figure figure (nun-None, figsize= $(5,2)$, dpi=80, facecolor=' $w^{\prime}$, edgecolor=' $k$ ') autocorrelation_plot(rainfall) autocorrelation
pyplot. show() 


\section{Source Code for Complete Time Series Analysis Run}

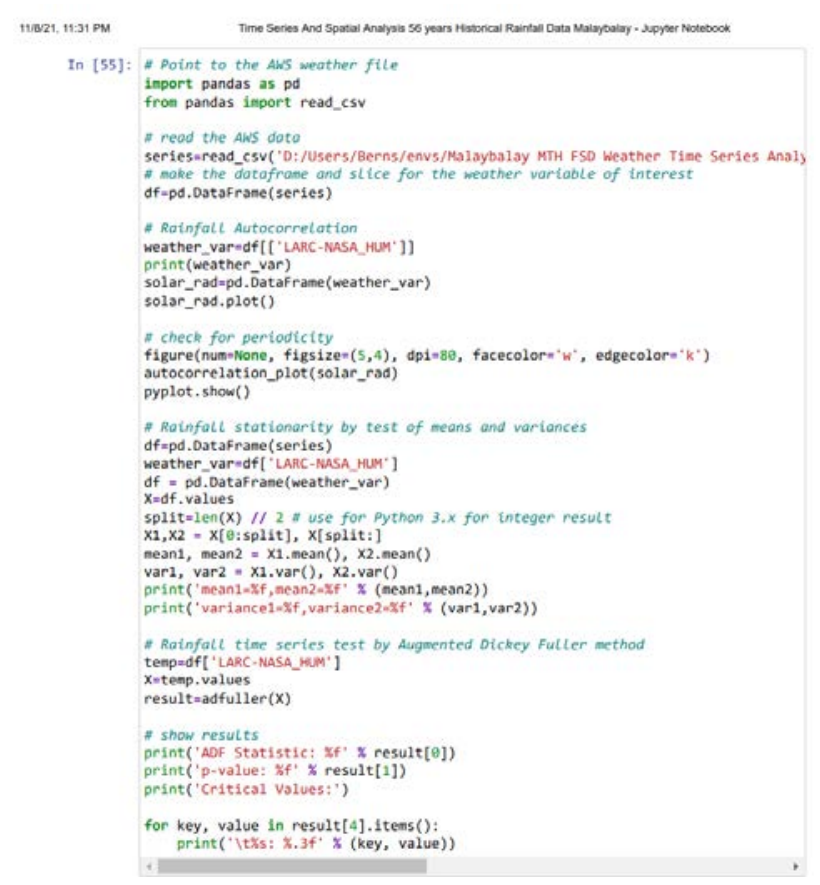

